\title{
ON SOME SPECTRAL PROPERTIES OF ELLIPTIC PSEUDODIFFERENTIAL OPERATORS
}

\author{
M. W. WONG
}

\begin{abstract}
We prove that the minimal and maximal operators associated with an elliptic pseudodifferential operator coincide in $L^{p}\left(\mathbf{R}^{n}\right), 1<p<\infty$. We obtain a set of necessary and sufficient conditions for a measurable function $q$ on $\mathbf{R}^{n}$ to be compact relative to some integral power of a constant coefficient elliptic pseudodifferential operator.
\end{abstract}

1. Introduction. For $m \in(-\infty, \infty)$, we denote by $S^{m}$ the set of all functions $\sigma$ in $C^{\infty}\left(\mathbf{R}^{n} \times \mathbf{R}^{n}\right)$ such that, for all multi-indices $\alpha$ and $\beta$, there exists a constant $C_{\alpha \beta}>0$ satisfying

$$
\left|\left(D_{x}^{\alpha} D_{\xi}^{\beta} \sigma\right)(x, \xi)\right| \leq C_{\alpha \beta}(1+|\xi|)^{m-|\beta|}
$$

for all $x, \xi$ in $\mathbf{R}^{n}$. Let $\sigma \in S^{m}$. Then we define the operator $T_{\sigma}$ on $S$ (the Schwartz space) by

$$
\left(T_{\sigma} \phi\right)(x)=(2 \pi)^{-n} \int_{\mathbf{R}^{n}} e^{i x \cdot \xi} \sigma(x, \xi) \hat{\phi}(\xi) d \xi
$$

for all $\phi$ in $S$, where

$$
\hat{\phi}(\xi)=\int_{\mathbf{R}^{n}} e^{-i x \cdot \xi} \phi(x) d x
$$

We call $T_{\sigma}$ a pseudodifferential operator with symbol $\sigma$ in $S^{m}$. The smallest number $m$ for which $\sigma \in S^{m}$ is called the order of $\sigma$ (or $T_{\sigma}$ ). Furthermore, we call $\sigma$ an elliptic symbol or $T_{\sigma}$ an elliptic pseudodifferential operator if there exist positive constants $C$ and $R$ such that

$$
|\sigma(x, \xi)| \geq C(1+|\xi|)^{m}
$$

for $|\xi| \geq R$.

The aim of this paper is to study some spectral properties of elliptic pseudodifferential operators on $L^{p}\left(\mathbf{R}^{n}\right), 1<p<\infty$. In $\S 2$, we recall some standard results of pseudodifferential operators and derive some easy consequences which we shall use afterwards. In $\S 3$, we prove that the minimal and maximal operators asociated with an elliptic pseudodifferential operator with symbol in $S^{m}$ coincide in $L^{p}\left(\mathbf{R}^{n}\right), 1<p<\infty$. This result can be generalized to a wider class of pseudodifferential operators with symbols in $S_{\rho, \delta}^{m}$. See the monograph [3] by Kumano-go for a comprehensive account of these operators. If $\sigma \in S^{m}$ is any symbol independent of $x$, then it follows from Theorem 2.4 in Wong [7] that the minimal and maximal operators associated with $T_{\sigma}$ coincide in $L^{p}\left(\mathbf{R}^{n}\right), 1 \leq p<\infty$. In $\S 4$ and 5 ,

Received by the editors February 5, 1986.

1980 Mathematics Subject Classification (1985 Revision). Primary 47G05, 35 S05.

Key words and phrases. Elliptic pseudodifferential operators, minimal operators, maximal operators, relative compactness. 
we study operators of the form $T_{\sigma}^{k}+q$, where $\sigma$ is an elliptic symbol of positive order $m, T_{\sigma}^{k}$ the $k$ th power of $T_{\sigma}$ and $q$ a measurable function on $\mathbf{R}^{n}$. Such operators come up naturally in quantum physics. The most celebrated one has symbol $\tau(\xi)=\sqrt{|\xi|^{2}+\mu^{2}}$. The operator $T_{\tau}+q$ is reminiscent of the relativistic Hamiltonian of a spinless particle of mass $\mu$ under the influence of the potential $q$. A main result in the paper is a characterization of functions $q$ which are compact relative to some power of $T_{\sigma}$, where $\sigma$ is an elliptic symbol of positive order and independent of $x$ in $\mathbf{R}^{n}$. The special case when $T_{\sigma}$ is a constant coefficient ordinary differential operator can be found in $\S 9$ of Chapter 14 in Schechter [6]. For the connection between relative compactness and the invariance of the essential spectrum, see Gustafson and Weidmann [2], and Schechter [4].

Finally, it should be pointed out that Theorems 4.1 and 5.1 in this paper are in fact equivalent to the corresponding results obtained by Balslev in $[\mathbf{1}]$ for elliptic partial differential operators with constant coefficients. However, an approach via pseudodifferential operators is perhaps also enlightening.

2. Some fundamental results. We recall some fundamental properties of pseudodifferential operators.

THEOREM 2.1. Let $\sigma_{1} \in S^{m_{1}}$ and $\sigma_{2} \in S^{m_{2}}$. Then the composition $T_{\sigma_{1}} T_{\sigma_{2}}$ is a pseudodifferential operator with symbol $\sigma$ in $S^{m_{1}+m_{2}}$. Moreover, $\sigma(x, \xi)$ has the asymptotic expansion

$$
\sigma(x, \xi) \sim \sum_{\alpha} \frac{(-i)^{|\alpha|}}{\alpha !}\left[\partial_{\xi}^{\alpha} \sigma_{1}\right](x, \xi)\left[\partial_{x}^{\alpha} \sigma_{2}\right](x, \xi) .
$$

Here, formula (2.1) means that

$$
\sigma(x, \xi)-\sum_{|\alpha|<N} \frac{(-i)^{|\alpha|}}{\alpha !}\left[\partial_{\xi}^{\alpha} \sigma_{1}\right](x, \xi)\left[\partial_{x}^{\alpha} \sigma_{2}\right](x, \xi)
$$

is a symbol in $S^{m_{1}+m_{2}-N}$ for every positive integer $N$.

THEOREM 2.2. Let $\sigma \in S^{m}$. Then the formal adjoint $T_{\sigma}^{*}$ is a pseudodifferential operator with symbol $\sigma^{*}$ in $S^{m}$. Moreover, $\sigma^{*}(x, \xi)$ has the asymptotic expansion

$$
\sigma^{*}(x, \xi) \sim \sum_{\alpha} \frac{(-i)^{|\alpha|}}{\alpha !} \overline{\left[\partial_{x}^{\alpha} \partial_{\xi}^{\alpha} \sigma\right](x, \xi)} .
$$

Here, formula (2.2) means that

$$
\sigma^{*}-\sum_{|\alpha|<N} \frac{(-i)^{|\alpha|}}{\alpha !} \overline{\left[\partial_{x}^{\alpha} \partial_{\xi}^{\alpha} \sigma\right]}
$$

is a symbol in $S^{m-N}$ for every positive integer $N$.

Theorems 2.1 and 2.2 are special cases of Theorem 1.7, pp. 59-60 of Kumano-go $[3]$.

As an application of Theorems 2.1 and 2.2, we give the following theorem. 
THEOREM 2.3. Let $T_{\sigma_{1}}$ and $T_{\sigma_{2}}$ be elliptic pseudodifferential operators of orders $m_{1}$ and $m_{1}$ respectively. Then $T_{\sigma_{1}} T_{\sigma_{2}}$ and $T_{\sigma_{1}}^{*}$ are elliptic pseudodifferential operators of orders $m_{1}+m_{2}$ and $m_{1}$ respectively.

ProOF. Since $T_{\sigma_{1}}$ and $T_{\sigma_{2}}$ are elliptic, we can find positive constants $C_{1}, C_{2}$, and $R_{1}$ such that

$$
\begin{aligned}
|\sigma(x, \xi)| & \geq\left|\sigma_{1}(x, \xi) \sigma_{2}(x, \xi)\right|-\left|\sigma(x, \xi)-\sigma_{1}(x, \xi) \sigma_{2}(x, \xi)\right| \\
& \geq C_{1}(1+|\xi|)^{m_{1}+m_{2}}-C_{2}(1+|\xi|)^{m_{1}+m_{2}-1} \\
& =(1+|\xi|)^{m_{1}+m_{2}}\left\{C_{1}-C_{2}(1+|\xi|)^{-1}\right\}, \quad|\xi| \geq R_{1} .
\end{aligned}
$$

Now we can choose $|\xi|$ large enough, say $|\xi| \geq R_{2}$, so that $C_{1}-C_{2}(1+|\xi|)^{-1} \geq \frac{1}{2} C_{1}$. Hence,

$$
|\sigma(x, \xi)| \geq \frac{1}{2} C_{1}(1+|\xi|)^{m_{1}+m_{2}}
$$

for $|\xi| \geq R$, where $R=\max \left(R_{1}, R_{2}\right)$. This proves that $T_{\sigma_{1}} T_{\sigma_{2}}$ is elliptic. The proof of the ellipticity of $T_{\sigma_{1}}^{*}$ is similar and hence omitted.

The formal adjoint allows us to extend the definition of $T_{\sigma}$ from $S$ to the conjugate linear dual $S^{*}$ of $S$, i.e., the space of tempered distributions. To wit, take any $u \in S^{*}$ and we can define $T_{\sigma} u$ by

$$
\left(T_{\sigma} u\right)(\phi)=u\left(T_{\sigma}^{*} \phi\right)
$$

for all $\phi$ in $S$. A very important result is the following theorem.

THEOREM 2.4. Let $\sigma \in S^{m}$. Then $T_{\sigma}: H^{s+m, p} \rightarrow H^{s, p}$ is a bounded linear operator for $-\infty<s<\infty$ and $1<p<\infty$.

$H^{s, p}$ is the $L^{p}$ Sobolev space of order $s$. See Chapter 2, $\S 4$ of Schechter [5] for a discussion of these spaces. For a proof of Theorem 2.4, take $\omega(x) \equiv 1$ in Theorem 2.1 of Wong [8].

3. The minimal and maximal operators. Let $\sigma \in S^{m}$. Then $T_{\sigma}$ can be considered as an operator on $L^{p}\left(\mathbf{R}^{n}\right)$ with domain $S$. However, it is not closed. Fortunately, it is closable. Hence, it has a closed extension. See Chapter $12, \S 6$ of Schechter [6].

Proposition 3.1. The operator $T_{\sigma}$ defined on $L^{p}\left(\mathbf{R}^{n}\right)$ with domain $S$ is closable.

ProOF. Let $\left\{\phi_{k}\right\}$ be a sequence of functions $S$ such that $\phi_{k} \rightarrow 0, T_{\sigma} \phi_{k} \rightarrow f$ in $L^{p}\left(\mathbf{R}^{n}\right)$ as $k \rightarrow \infty$. Then for any function $\psi$ in $S$, we have

$$
\left(T_{\sigma} \phi_{k}, \psi\right)=\left(\phi_{k}, T_{\sigma}^{*} \psi\right)
$$

where $T_{\sigma}^{*}$ is the formal adjoint constructed in Theorem 2.2. Let $k \rightarrow \infty$. Then we have $(f, \psi)=0$ for all functions $\psi$ in $S$. Since $S$ is dense in $L^{p}\left(\mathbf{R}^{n}\right)$, it follows that $f=0$.

REMARK 3.2. A consequence of Proposition 3.1 is that $T_{\sigma}: S \rightarrow L^{p}\left(\mathbf{R}^{n}\right)$ has a closed extension in $L^{p}\left(\mathbf{R}^{n}\right)$. We denote the smallest such by $T_{\sigma, \min }$ and call it the minimal operator of $T_{\sigma}$. It can be shown easily that the domain $D\left(T_{\sigma, \min }\right)$ of $T_{\sigma, \min }$ consists of all functions $u$ in $L^{p}\left(\mathbf{R}^{n}\right)$ for which a sequence $\left\{\phi_{k}\right\}$ in $S$ can be found such that $\phi_{k} \rightarrow u$ in $L^{p}\left(\mathbf{R}^{n}\right)$ and $T_{\sigma} \phi_{k} \rightarrow f$ for some $f$ in $L^{p}\left(\mathbf{R}^{n}\right)$. Moreover, $T_{\sigma, \min } u=f$. See again $[6$, Chapter $12, \S 6]$. 
We say that a function $u$ in $L^{p}\left(\mathbf{R}^{n}\right)$ lies in $D\left(T_{\sigma, \max }\right)$ and $T_{\sigma, \max } u=f$ if and only if

$$
\left(u, T_{\sigma}^{*} \phi\right)=(f, \phi)
$$

for all $\phi$ in $S$.

REMARK 3.3 . It can be shown easily that $T_{\sigma, \max }$ is a closed linear operator on $L^{p}\left(\mathbf{R}^{n}\right)$ with domain $D\left(T_{\sigma, \max }\right)$ containing $S$. We call $T_{\sigma, \max }$ the maximal operator of $T_{\sigma}$. It is clear that $D\left(T_{\sigma, \max }\right)$ consists of all functions $u$ in $L^{p}\left(\mathbf{R}^{n}\right)$ for which $T_{\sigma} u$ is in $L^{p}\left(\mathbf{R}^{n}\right)$. Furthermore, $T_{\sigma, \max } u=T_{\sigma} u$.

The main result in this section is that $T_{\sigma, \min }=T_{\sigma, \max }$ if $\sigma$ is elliptic. To this end, we recall a very important result in the theory of pseudodifferential operators.

THEOREM 3.4. Let $\sigma$ be an elliptic symbol of order $m$. Then $T_{\sigma}$ has a parametrix $T_{\tau}$ with $\tau \in S^{-m}$. More precisely,

$$
T_{\tau} T_{\sigma}=I+R, \quad T_{\sigma} T_{\tau}=I+S
$$

where $I$ is the identity, $R$ and $S$ are pseudodifferential operators with symbols in $\bigcap_{k=-\infty}^{\infty} S^{k}$.

For a proof of Theorem 3.4, see Kumano-go [3, pp. 83-85].

We can now give more precise descriptions of $T_{\sigma, \min }$ and $T_{\sigma, \max }$ when $\sigma$ is elliptic. First, we consider $T_{\sigma, \min }$.

THEOREM 3.5. Let $\sigma$ be an elliptic symbol of order $m$. Then $D\left(T_{\sigma, \min }\right)=H^{m, p}$ and $T_{\sigma, \min } u=T_{\sigma} u$ for all $u$ in $H^{m, p}$.

PROOF. The theorem follows easily from the estimate

$$
\|\phi\|_{m, p} \leq C\left(\left\|T_{\sigma} \phi\right\|_{0, p}+\|\phi\|_{0, p}\right) \leq C^{\prime}\|\phi\|_{m, p}, \quad \phi \in S .
$$

Indeed, if $u \in H^{m, p}$, then we can take a sequence $\left\{\phi_{k}\right\}$ of functions in $S$ such that $\phi_{k} \rightarrow u$ in $H^{m, p}$. Hence, by (3.2), $\left\{T_{\sigma} \phi_{k}\right\}$ and $\left\{\phi_{k}\right\}$ are Cauchy sequences in $L^{p}\left(\mathbf{R}^{n}\right)$. So $\phi_{k} \rightarrow u$ and $T_{\sigma} \phi_{k} \rightarrow f$ for some $u$ and $f$ in $L^{p}\left(\mathbf{R}^{n}\right)$. Hence $u \in D\left(T_{\sigma, \min }\right)$ and $T_{\sigma, \min } u=f$. On the other hand, if $u \in D\left(T_{\sigma, \min }\right)$, then we can find a sequence $\left\{\phi_{k}\right\}$ in $S$ such that $\phi_{k} \rightarrow u$ in $L^{p}\left(\mathbf{R}^{n}\right)$ and $T_{\sigma} \phi_{k} \rightarrow f$ for some $f$ in $L^{p}\left(\mathbf{R}^{n}\right)$. Hence, $\left\{\phi_{k}\right\}$ and $\left\{T_{\sigma} \phi_{k}\right\}$ are Cauchy sequences in $L^{p}\left(\mathbf{R}^{n}\right)$. So, by (3.2), $\left\{\phi_{k}\right\}$ is a Cauchy sequence in $H^{m, p}$. Since $H^{m, p}$ is complete, $\phi_{k} \rightarrow v$ for some $v$ in $H^{m, p}$. Suppose $m \geq 0$. Then the inclusion map $H^{m, p} \rightarrow L^{p}\left(\mathbf{R}^{n}\right)$ is continuous. Thus, $\phi_{k} \rightarrow v$ in $L^{p}\left(\mathbf{R}^{n}\right)$. Hence $u=v$, and consequently lies in $H^{m, p}$. If $m<0$, then the inclusion map $L^{p}\left(\mathbf{R}^{n}\right) \rightarrow H^{m, p}$ is continuous. Thus, $\phi_{k} \rightarrow u$ in $H^{m, p}$. Hence $u=v$, and consequently lies in $H^{m, p}$ as well. It remains to prove the inequalities (3.2). First, note that the second inequality follows from Theorem 2.4. To prove the first inequality, note that (3.1) implies that

$$
\phi=T_{\tau} T_{\sigma} \phi-R \phi
$$

for all $\phi$ in $S$. Since $\tau \in S^{-m}$ and $R$ has symbol in $S^{-m}$, it follows from Theorem 2.4 again that

$$
\|\phi\|_{m, p} \leq C\left(\left\|T_{\sigma} \phi\right\|_{0, p}+\|\phi\|_{0, p}\right)
$$

for all $\phi$ in $S$.

We can now prove the following result. 
THEOREM 3.6. Let $\sigma$ be an elliptic symbol of order $m$. Then $T_{\sigma, \min }=T_{\sigma, \max }$.

Proof. Since $T_{\sigma, \min }$ is the smallest closed extension of $T_{\sigma}: S \rightarrow L^{p}\left(\mathbf{R}^{n}\right)$ in $L^{p}\left(\mathbf{R}^{n}\right)$, it follows from Theorem 3.5 that it is sufficient to prove that $D\left(T_{\sigma, \max }\right)=$ $H^{m, p}$. Obviously, $H^{m, p} \subseteq D\left(T_{\sigma, \max }\right)$ by Remark 3.3. To prove the other inclusion, let $u \in D\left(T_{\sigma, \max }\right)$. Then by (3.1), $u=T_{\tau} T_{\sigma} u-R u$. Since $\tau \in S^{-m}$ and $T_{\sigma} u \in$ $L^{p}\left(\mathbf{R}^{n}\right)$, we have $T_{\tau} T_{\sigma} u \in H^{m, p}$. Since $R$ has symbol in $S^{-m}$ and $u \in L^{p}\left(\mathbf{R}^{n}\right)$, we also have $R u \in H^{m, p}$. Hence, $u \in H^{m, p}$.

4. Relative compactness. Let $q$ be a measurable function defined on $\mathbf{R}^{n}$. Denote by $D(q)$ the set of all functions $u$ in $L^{p}\left(\mathbf{R}^{n}\right)$ for which $q u$ is also in $L^{p}\left(\mathbf{R}^{n}\right)$. Then it can be shown easily that the multiplication operator $q: D(q) \rightarrow L^{p}\left(\mathbf{R}^{n}\right)$ given by $u \rightarrow q u$ is a closed operator in $L^{p}\left(\mathbf{R}^{n}\right)$.

For any elliptic symbol $\sigma$ of positive order $m$, we know from Theorem 2.3 that $T_{\sigma}^{k}$ is an elliptic pseudodifferential operator of order $\mathrm{km}$ for any positive integer $k$. We say that $q$ is $T_{\sigma}^{k}$ compact if and only if $H^{k m, p} \subseteq D(q)$ and the multiplication operator $q: H^{k m, p} \rightarrow L^{p}\left(\mathbf{R}^{n}\right)$ is a compact operator. Using Theorems 2.1 and 4.1 in Chapter 6 of Schechter [5], we have the following result.

THEOREM 4.1. Let $T_{\sigma}$ be an elliptic pseudodifferential operator of positive order $m$ and $q$ a measurable function on $\mathbf{R}^{n}$. Then, in order for $q$ to be $T_{\sigma}^{k}$ compact for some $k \geq 1$, it is sufficient that

$$
\sup _{y} \int_{|x-y|<1}|q(x)|^{p} d x<\infty
$$

and

$$
\lim _{|y| \rightarrow \infty} \int_{|x-y|<1}|q(x)|^{p} d x=0 .
$$

We shall prove in $\S 5$ that these conditions are also necessary when the symbol $\sigma$ is independent of $x$.

5. Schrödinger-type operators. Let $m \in(-\infty, \infty)$. We denote by $S_{0}^{m}$ the set of all $C^{\infty}$ functions $\sigma$ on $\mathbf{R}^{n}$ such that for any mutli-index $\alpha$, there exists a constant $C_{\alpha}>0$ satisfying

$$
\left|\left(D^{\alpha} \sigma\right)(\xi)\right| \leq C_{\alpha}(1+|\xi|)^{m-|\alpha|}
$$

for all $\xi \in \mathbf{R}^{n}$. Obviously, $S_{0}^{m}$ is a subset of $S^{m}$. Hence, everything we have stated for $S^{m}$ is also valid for $S_{0}^{m}$.

In this section, we are mainly interested in operators of the form $T_{\sigma}^{k}+q$, where $\sigma \in S_{0}^{m}$ is elliptic of positive order $m, k$ is a positive integer, and $q$ is a measurabłe function on $\mathbf{R}^{n}$. We call such operators Schrödinger-type operators. See the Introduction (i.e., §1) for the importance of these operators.

THEOREM 5.1. Let $T_{\sigma}$ be an elliptic pseudodifferential operator with symbol in $S_{0}^{m}, m>0$; and let $q$ be a measurable function on $\mathbf{R}^{n}$. Then in order for $q$ to be compact relative to some positive integral power $T_{\sigma}^{k}$ of $T_{\sigma}$, it is necessary and sufficient that (4.1) and (4.2) are valid.

ProOF. Of course, in view of Theorem 4.1, we need only prove necessity. Since $\sigma \in S_{0}^{m}$, it follows that $T_{\sigma}^{k}=T_{\tau}$, where $\tau(\xi)=\sigma(\xi)^{k}$. If $q$ is compact relative to $T_{\tau}$, 
then $H^{k m, p} \subseteq D(q)$ and $q: H^{k m, p} \rightarrow L^{p}\left(\mathbf{R}^{n}\right)$ is compact, hence bounded. Since, by (3.2), $\|\phi\|_{k m, p}$ is equivalent to $\left\|T_{\tau} \phi\right\|_{0, p}+\|\phi\|_{0, p}$, we can find a constant $C>0$ such that

$$
\|q \phi\|_{0, p} \leq C\left(\left\|T_{\tau} \phi\right\|_{0, p}+\|\phi\|_{0, p}\right)
$$

for all $\phi$ in $S$. Let $\psi \in C_{0}^{\infty}\left(\mathbf{R}^{n}\right)$ be such that $\psi(x) \geq 0$ for all $x$ in $\mathbf{R}^{n}$, and $\psi(x) \geq 1$ for $|x| \leq 1$. Define $\psi_{y}$ by $\psi_{y}(x)=\psi(x-y)$, for any fixed but arbitrary $y$ in $\mathbf{R}^{n}$. Then $\psi_{y} \in C_{0}^{\infty}\left(\mathbf{R}^{n}\right)$ and an easy computation shows that

$$
\left(T_{\tau} \psi_{y}\right)(x)=\left(T_{\tau} \psi\right)(x-y)
$$

Hence, by (5.1)

$$
\begin{aligned}
\left\|q \psi_{y}\right\|_{0, p} & \leq C\left(\left\|T_{\tau} \psi_{y}\right\|_{0, p}+\left\|\psi_{y}\right\|_{0, p}\right) \\
& =C\left(\left\|T_{\tau} \psi\right\|_{0, p}+\|\psi\|_{0, p}\right)
\end{aligned}
$$

But,

$$
\left\|q \psi_{y}\right\|_{0, p} \geq\left\{\int_{|x-y|<1}\left|q(x) \psi_{y}(x)\right|^{p} d x\right\}^{1 / p} \geq\left\{\int_{|x-y|<1}|q(x)|^{p} d x\right\}^{1 / p} .
$$

Hence, by (5.3), (4.1) is proved. To prove (4.2), suppose that it is not true. Then there exists a $\delta>0$ such that

$$
\int_{\left|x-y_{j}\right|<1}|q(x)|^{p} d x>\delta
$$

for some sequence $\left\{y_{j}\right\}$ in $\mathbf{R}^{n}$. Let $\psi$ be as before and define $\psi_{j}$ by $\psi_{j}(x)=\psi\left(x-y_{j}\right)$ for $j=1,2, \ldots$ Then by $(3.2)$ and (5.2),

$$
\begin{aligned}
\left\|\psi_{j}\right\|_{k m, p} & \sim\left\|\psi_{j}\right\|_{0, p}+\left\|T_{\tau} \psi_{j}\right\|_{0, p} \\
& =\|\psi\|_{0, p}+\left\|T_{\tau} \psi\right\|_{0, p} .
\end{aligned}
$$

Since $q: H^{k m, p} \rightarrow L^{p}\left(\mathbf{R}^{n}\right)$ is compact, it follows that there exists a subsequence of $\left\{\psi_{j}\right\}$, again denoted by $\left\{\psi_{j}\right\}$, such that $q \psi_{j} \rightarrow f$, for some $f$ in $L^{p}\left(\mathbf{R}^{n}\right)$. But,

$$
\int_{\mathbf{R}^{n}}\left|q(x) \psi_{j}(x)\right|^{p} d x \geq \int_{\left|x-y_{j}\right|<1}|q(x)|^{p} d x \geq \delta
$$

for $j=1,2, \ldots$ Hence,

$$
\|f\|_{0, p} \geq \delta
$$

But on any unit cube $Q$ in $\mathbf{R}^{n}, \psi_{j}(x)=0$ if $j$ is large enough. So, $q(x) \psi_{j}(x)=0$ for any $x$ in $Q$. Hence, $\lim _{j \rightarrow \infty} \int_{Q}\left|q(x) \psi_{j}(x)\right|^{p} d x=0$, and consequently, $\int_{Q}|f(x)|^{p} d x$ $=0$. By partitioning $\mathbf{R}^{n}$ into a grid of unit cubes, we see that $\int_{\mathbf{R}^{n}}|f(x)|^{p} d x=0$. This contradicts (5.4).

ACKNOWLEDGMENT. This resarch was partially supported by the Natural Sciences and Engineering Research Council of Canada under Grant No. A8562. 


\section{REFERENCES}

1. E. Balslev, The essential spectrum of elliptic operators in $L^{p}\left(\mathbf{R}^{n}\right)$, Trans. Amer. Math. Soc. 116 (1965), 193-217.

2. K. Gustafson and J. Weidmann, On the essential spectrum, J. Math. Anal. Appl. 25 (1969), 121-127.

3. H. Kumano-go, Psuedo-differential operators, MIT Press, 1981.

4. M. Schechter, On the essential spectrum of an arbitrary operator. I, J. Math. Anal. Appl. 13 (1966), 205-215.

5. __ Spectra of partial differential operators, North-Holland, Amsterdam, 1971.

6. __ Principles of functional analysis, Academic Press, New York, 1971.

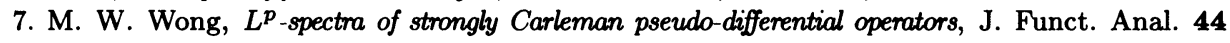
(1981), 163-173.

8 M. W. Wong, Fredholm pseudo-differential operators on weighted Sobolev spaces, Ark. Mat. 21 (1983), 271-282.

Department of MAThematics, York University, 4700 KeEle Steet, North YORK, ONTARIO, CANADA M3J 1P3 